\title{
Utilização do método ELECTRE 11 para avaliação de pilotos no campeonato de Fórmula 1
}

\author{
Maria Cecília de Carvalho Chaves ${ }^{\mathrm{a}, *}$, Silvio Figueiredo Gomes Júnior ${ }^{\mathrm{b}}$, \\ Eliane Ribeiro Pereirac ${ }^{\mathrm{c}}$, João Carlos Correia Baptista Soares de Mello ${ }^{\mathrm{d}}$ \\ a,*mariaceci@hotmail.com, UFF, Brasil \\ bsilviofgj@gmail.com, UFF, Brasil \\ 'egr1@globo.com, UFF, Brasil \\ djcsmello@producao.uff.br, UFF, Brasil
}

\begin{abstract}
Resumo
Escolher um bom piloto é tarefa difícil, que ultrapassa a identificação de habilidade e coragem para pilotar automóveis. Pilotos que não disputam a liderança das competições costumam receber pouco destaque porque as transmissões esportivas enfatizam a luta pelas melhores posições. A busca por pilotos tem importância especial para equipes menores, incapazes de pagar altos salários. Identificar um bom piloto pode representar ganhos significativos, permitindo à equipe obter melhor desempenho no campeonato. Este artigo propõe uma forma de avaliação desses pilotos no campeonato de F1, usando o método de apoio à decisão multicritério ELECTRE 11.0 objetivo é propor uma ordenação de pilotos, a fim de identificar os mais indicados para serem contratados por equipes pequenas/ médias, proporcionando-lhes maior competitividade na temporada seguinte. A análise foi elaborada considerando-se os pilotos que participaram da temporada de 2007.
\end{abstract}

Palavras-chave

ELECTRE 11. Multicritério. Fórmula 1.

\section{Introdução}

Um problema frequentemente enfrentado pelos chefes das escuderias pequenas e médias de Fórmula 1 (F1) é a escolha do piloto que integrará a equipe na temporada vindoura visando ter maior competitividade. Qualquer melhora adicional que possa ser obtida por estas equipes pode trazer novos patrocínios e outras vantagens econômicas relevantes para a escuderia. Um bom piloto é capaz de trazer não apenas melhores colocações para a escuderia, mas também um maior desenvolvimento para os carros. Isto é particularmente crítico nas equipes pequenas e médias, por apresentarem orçamentos limitados, possuindo, portanto, menor potencial para contratar pilotos consagrados. A consideração de mais de um ponto de vista para estabelecer o que venha a ser um "bom" piloto torna o problema multicritério.

Este artigo é uma evolução do trabalho apresentado por Gomes Júnior e Soares e Mello (2007a). 0 propósito aqui não é mais o de estabelecer uma ordenação para os pilotos no campeonato, mas o de identificar, com o apoio de uma ferramenta multicritério, pilotos que atendam às reais necessidades de equipes de $\mathrm{F} 1$ pequenas $\mathrm{e}$ médias, com capacidade de desenvolver os carros dessas equipes, alavancando o desenvolvimento delas.

A proposta deste trabalho é utilizar um método de apoio à decisão multicritério não compensatório para propor uma ordenação de pilotos que identifique os mais indicados para serem contratados por equipes pequenas/médias de forma a garantirlhes maior competitividade.

Nos últimos anos, foram apresentadas na literatura diversas aplicações da Pesquisa Operacional (P0) em esportes. No futebol, as aplicações foram inúmeras. Dixon e Coles (1997) utilizaram a PO para estabelecer comparações entre equipes inglesas 
de futebol entre 1992 e 1995. Dixon e Robinson (1998) descreveram a importância do futebol para a Inglaterra e criaram um modelo para analisar resultados de jogos e estabelecer critérios para apostas. Koning (2000) comparou os resultados dos jogos do futebol holandês desde a década de 1970. Held e Vollnhals (2005) utilizaram a PO para estabelecer comparações entre equipes participantes dos maiores campeonatos de futebol da Europa e propuseram uma nova forma de estabelecer o "peso" que determina o número de equipes que participam das ligas européias estudadas.

Além de aplicações no futebol, Pollard (2002) verificou as alterações ocorridas entre o público espectador dos jogos de baseball e hockey no gelo, nos Estados Unidos, quando a equipe troca o estádio antigo por um novo. Holder e Nevill (1997) compararam o desempenho de jogadores de tênis disputando partidas dentro e fora do seu país.

Em relação à utilização da $\mathrm{PO}$ nas competições de Fórmula 1, Kladroba (2000) discutiu critérios para o estabelecimento de rankings e utilizou como exemplo os resultados do campeonato de 1998; já Soares de Mello et al. (2005a) utilizaram métodos multicritério para estabelecer a ordenação dos pilotos no campeonato de 2002, enquanto Gomes Júnior e Soares de Mello (2007b) usaram modelos DEA para avaliar pilotos e equipes no Campeonato Mundial de Fórmula 1 de 2006. A questão do sistema de ordenação usado nas Olimpíadas também tem sido estudada por alguns autores: Churilov e Flitman (2006) sugeriram o uso de DEA para a obtenção de um sistema classificatório que fosse considerado "justo" pela maior parte dos países participantes; lins et al. (2003) discutiram aspectos teóricos do uso do modelo DEA-GSZ (Ganhos de Soma Zero) na mensuração da performance dos países nos jogos olímpicos de verão e Soares de Mello et al. (2008) e Soares de Mello, Angulo Meza e Branco da Silva (2009) utilizaram modelos DEA com input unitário e avaliação cruzada.

Este trabalho está organizado da seguinte forma: no item 2 discute-se o atual sistema utilizado na F1. Em seguida é apresentada uma breve descrição dos métodos de classificação. A seção 4 apresenta um estudo de caso - o Campeonato de F1 de 2007, em que o ELECTRE 11 é aplicado. No item 5 faz-se uma análise dos resultados obtidos e uma comparação com o método ordinal de Condorcet e, por fim, seguem-se as conclusões e as principais contribuições.

\section{0 campeonato mundial de Fórmula 1}

0 Campeonato de Fórmula 1, originário das competições de Grandes Prêmios (GPs) iniciadas antes mesmo da Segunda Guerra Mundial, começou em 1950, na Inglaterra. Inicialmente, os GPs aconteciam apenas na Europa e o status mundial era garantido pela inclusão do resultado das 500 milhas de Indianópolis, mesmo sendo as equipes e pilotos dessa prova totalmente distintos dos que disputavam as ocorridas na Europa, como descrito em Gomes Júnior (2006).

No início, os carros utilizados não eram totalmente padronizados, como os atuais. Com o tempo, grandes equipes foram aderindo ao campeonato, garantindo-lhe prestígio crescente. Ao longo dos anos, as regras e as normas, principalmente as relativas à segurança - para o piloto e para o público -, foram evoluindo, mantendo-se, no entanto, a preocupação com a permanência de elementos fundamentais para os amantes do esporte: velocidade, emoção, competição.

As principais e mais profundas mudanças da F1 ocorreram na década de 60. Iniciou-se um processo de profunda reformulação dos carros, que passaram a ter motor traseiro, chassi monocoque e aerodinâmica arrojada; os pneus passaram a ser mais largos, para logo depois, em 1970, surgirem os primeiros pneus slick - pneus sem frisos, que garantem maior aderência dos carros à pista, aumentando sua velocidade - Jenkins e Floyd (2001). Outra importante mudança foi decisiva para a divulgação e crescimento do esporte: a equipe Lotus iniciou uma parceria com uma grande empresa de outro segmento (tabaco) e seu carro ganhou novas cores, inaugurando uma fase comercial para o esporte, com a entrada dos patrocinadores na categoria. Até 1968, as equipes de F1 eram mantidas com a ajuda das empresas de petróleo e de fabricantes de pneus, aporte pequeno se comparado às relações comerciais dos dias atuais, quando o esporte envolve quantias extremamente elevadas e interesses que ultrapassam as fronteiras da simples competição esportiva.

$\mathrm{Na}$ década de 70 foram modificadas as vantagens comerciais dos donos dos circuitos, o que garantiu maior autonomia para a FlA - Federação Internacional de Automobilismo, inclusive com relação às negociações dos direitos de transmissão de TV, o que passou a atrair patrocínios cada vez mais elevados. 
0 regulamento do Campeonato Mundial de Fórmula 1 determina que o campeão da temporada é o piloto que soma o maior número de pontos ao final de todas as corridas da temporada. Até 2003, os demais pilotos tinham a posição no campeonato determinada pelo total de pontos alcançados. A partir deste ano, em cada corrida, apenas os oito primeiros colocados passaram a somar pontos, sendo a pontuação de cada colocado apresentada na Tabela 1.

Este regulamento é uma variação do método de Borda (maiores detalhes em Barba-Romero e Pomerol (1997)). A diferença mais evidente em relação ao método de Borda tradicional é que os primeiros colocados marcam mais pontos, enquanto que com o uso do método original marcariam menos.

0 regulamento prevê ainda a possibilidade de empate na pontuação final, preconizando sucessivos critérios de desempate. Assim, as regras usam, na verdade, o método Lexicográfico.

A posição do piloto e da equipe determinadas por estes métodos no final da temporada é fator fundamental para a distribuição de verba da temporada seguinte. Por isso, o desenvolvimento de carros, chassis, enfim, a busca de melhorias que possam trazer alguma vantagem competitiva para as equipes são as maiores preocupações dos chefes de equipe. Todavia existe um elemento tão importante quanto a garantia de ter um bom carro e que não pode ser desprezado: um bom piloto.

A escolha de um bom piloto de F1 é tarefa difícil, que vai além da identificação de habilidade e coragem para dirigir carros tão velozes. Elementos como um bom preparo físico e psicológico, inteligência, determinação, dedicação, conhecimento de motores, além de grande paixão por velocidade $\mathrm{e}$ competições automobilísticas precisam fazer parte do perfil de um grande piloto.

É impossível não considerar os aspectos econômicos envolvidos nessa decisão. A contratação de um piloto no auge da carreira é impensável para escuderias menores, incapazes de pagar altos salários. Assim, para uma equipe pequena/média é importante buscar pilotos que não estejam no auge da carreira, ou seja, pilotos em início de carreira ou que já estejam próximos do seu término, pois estes, por não estarem em evidência na categoria, não percebem salários elevados. Entre os primeiros, ela pode encontrar um grande talento que a ajude, num tempo relativamente curto, a ganhar espaço nesse mundo altamente competitivo. Por sua vez, os pilotos com maior tempo de carreira têm a seu favor maior experiência, maior facilidade para ajustar os carros e conhecimento profundo de todos os elementos envolvidos na competição.

Por estes motivos, é preciso que se identifique entre os competidores um piloto cujo talento não seja reconhecido pelas equipes concorrentes - seja porque o piloto não tenha tido a oportunidade de dirigir um carro mais competitivo; porque ainda não tenha se adaptado totalmente à $\mathrm{F} 1$; ou porque, sendo muito jovem, não tenha ainda alcançado a maturidade necessária a um piloto dessa categoria; ou mesmo porque, ao longo de sua trajetória na $\mathrm{F} 1$, não tenha conseguido manter resultados que atendessem às expectativas das grandes equipes.

A busca por novos pilotos é, então, uma disputa à parte entre as equipes. Identificar um bom piloto a ser contratado pode representar ganhos significativos, pois possibilita o desenvolvimento dos carros e permite que a equipe realize os ajustes necessários para obter melhores resultados no campeonato e os benefícios correlatos.

Escuderias de pequeno/médio porte vivem uma realidade econômica pouco abastada e a obtenção de resultados positivos numa temporada podem representar uma perspectiva real de crescimento a curto prazo. Assim, este trabalho propõe o uso de um método de apoio à decisão multicritério não compensatório para auxiliar os chefes dessas equipes a identificar, dentre os pilotos do Campeonato de 2007, aqueles que, apesar de não serem muito valorizados pelos critérios adotados no campeonato, possam ser considerados para integrar a escuderia na temporada seguinte, proporcionando-lhe maior competitividade.

A realização deste estudo considerou a importância, para escuderias menores, de contratar um bom piloto num curto espaço de tempo, permitindo-lhe a conquista de benefícios capazes de alavancar seu crescimento. Assim, foram considerados para análise apenas os pilotos que participaram da temporada de 2007 da Fórmula 1 que têm a vantagem competitiva de já estarem adaptados a esta categoria.

Tabela 1. Pontuação da Fórmula 1 a partir de 2003.

\begin{tabular}{ccccccccc}
\hline Classificação & $1^{\circ}$ & $2^{\circ}$ & $3^{\circ}$ & $4^{\circ}$ & $5^{\circ}$ & $6^{\circ}$ & $7^{\circ}$ & $8^{\circ}$ \\
\hline Pontuação & 10 & 08 & 06 & 05 & 04 & 03 & 02 & 01 \\
\hline
\end{tabular}




\section{Métodos de apoio à decisão multicritério não compensatórios}

A existência de mais de um ponto de vista a ser considerado para a comparação entre um grupo de alternativas, ou, como é o caso deste estudo, a determinação da superioridade de uma sobre a outra pode ser uma tarefa bastante complexa, que demanda o uso de ferramentas capazes de resolver este tipo de questão.

$\mathrm{Na}$ análise multicritério são considerados diversos pontos de vista para avaliar o desempenho de cada alternativa com relação a cada um dos objetivos estabelecidos. De um modo geral, para que o objetivo final possa ser alcançado, é preciso que um fator relevante para a diferenciação das alternativas seja considerado, o que muitas vezes pode elevar o número de critérios a serem utilizados. Deve-se destacar que o valor do resultado final é sempre dependente dos critérios considerados e da metodologia escolhida, que, por sua vez, são sempre função do agente de decisão (AD), tornando a análise subjetiva.

0 método de Borda, considerado precursor dos métodos de agregação em uma única função síntese (função de utilidade multiatributo), efetua uma soma de pontos tendo, assim, a grande vantagem da simplicidade e, por isso, algumas de suas variantes são usadas em competições desportivas (SOARES DE MELLO, 2005a; KLADROBA, 2000). No entanto, apesar da simplicidade e do amplo uso de suas variações, o método de Borda não respeita um dos mais importantes axiomas de Arrow, o da independência em relação às alternativas irrelevantes. Ou seja, a posição final de duas alternativas não independe de sua classificação em relação a alternativas irrelevantes. Tal fato pode gerar distorções, com destaque para a extrema dependência dos resultados em referência ao conjunto de avaliação escolhido e para a possibilidade de manipulações pouco honestas.

0 método de Condorcet et al. apud Barba-Romero e Pomerol (1997), considerado precursor dos métodos seletivos (ou de sobreclassificação), trabalha com relações de superação. As alternativas são comparadas sempre duas a duas e constrói-se um grafo que expressa a relação entre elas. Este método, menos simples, tem a vantagem de impedir distorções ao fazer com que a posição relativa de duas alternativas independa de suas posições relativas a qualquer outra. No entanto, pode conduzir ao chamado paradoxo de Condorcet, ou situação de intransitividade. Isso acontece quando a alternativa $A$ supera a alternativa $B$, que supera a C, que, por sua vez, supera a alternativa A. Esta situação pode ser aproveitada em certos problemas, quando o objetivo é agrupar alternativas (SOARES DE MELLO, 2005a). No entanto, quando ocorre, impossibilita que seja gerada uma ordenação das alternativas.

Segundo Arrow (1951), Barba-Romero e Pomerol (1997), não existe método de escolha justo. Considera-se como justo um método de escolha multidecisor que obedeça aos axiomas de universalidade, de unanimidade, de independência em relação às alternativas irrelevantes, de transitividade e de totalidade. 0 teorema de Arrow garante que, com exceção de métodos de ditador, nenhum método de escolha atende simultaneamente a esses axiomas. 0 método de Copeland pode ser considerado um compromisso entre as filosofias opostas de Borda e Condorcet, reunindo, dentro do possível, as vantagens dos dois.

Gomes Júnior e Soares de Mello (2007) buscaram estabelecer uma ordenação para os pilotos no Campeonato de F1. 0 foco deste trabalho, que é um prolongamento deste último, é identificar, com o apoio de uma ferramenta multicritério, pilotos que atendam às reais necessidades de equipes de F1 pequenas/médias, provendo, assim, a escolha de pilotos que se enquadrem no perfil desejado.

A forma de explicitar as estruturas de preferência do decisor varia de acordo com o método de análise multicritério escolhido. Os chamados métodos ordinais são considerados bastante intuitivos e pouco exigentes tanto em termos computacionais quanto em relação às informações necessárias por parte do decisor. Alguns métodos buscam reproduzir a lógica compensatória e, para tal, fazem uso do modelo aditivo. Uma função de valor aditiva $v$ é a soma de funções unidimensionais, representando preferências sobre cada critério tomado separadamente. Para o uso de funções aditivas é condição necessária a existência de independência preferencial entre critérios. Em um primeiro momento, os métodos compensatórios parecem representar bem a estrutura utilizada nas competições desportivas.

Conforme Almeida e Costa (2003), a noção intuitiva de compensação sugere uma quantidade que contrabalance a desvantagem de um critério em relação a uma vantagem em outro. Os métodos não compensatórios requerem uma informação intercritério correspondente à importância relativa entre os critérios. Desta forma, evitam o favorecimento de ações que possuem um excelente desempenho em um critério mas que sejam fracas nos demais. 


\section{Estudo de caso: aplicação no Campeonato de 2007}

O Campeonato de 2007 contou com um total de 11 equipes de Fórmula 1, que disputaram 17 corridas, com 22 pilotos participando de cada prova. Como houve troca de pilotos durante a temporada, um total de 25 pilotos participaram. Entretanto, como o piloto Kazuki Nakajima participou apenas de uma corrida, no Grande Prêmio do Brasil, ele não foi considerado na análise.

Assumiu-se que o AD é um chefe de equipe fictício que precisa definir bons pilotos que possam ser contratados para sua escuderia, para a temporada seguinte, por terem tido bom desempenho durante a temporada atual, mas que não sejam valorizados pelos critérios de pontuação adotados na competição, ou seja, uma pessoa com grande conhecimento da categoria. Além disso, o AD opta por uma lógica não compensatória por esta ser mais coerente com os seus propósitos.

0 uso do método ELECTRE 11 justifica-se por ele ser não compensatório, o que exige pouco conhecimento do $\mathrm{AD}$, pelo fato de ser baseado em relações hierárquicas que possibilitam a adoção de critérios de natureza diferente como, por exemplo, quantitativos e qualitativos, que são utilizadas neste trabalho, além de o método ser um clássico e de fácil aplicação. Uma discussão atual acerca das interpretações da ordenação encontrada pelo ELECTRE 11 pode ser vista em Figueira e Roy (2009) e Wang (2008).

A relevância do trabalho consiste na criação de um modelo que permite apoiar os gestores na tomada de decisão que envolve diversos fatores, principalmente financeiros.

\subsection{O método ELECTRE II}

Os métodos da família ELECTRE, iniciados com a formulação do ELECTRE 1, apresentada em Roy (1968), foram desenvolvidos com o propósito de incorporar um caráter mais realista à tomada de decisão. Consideram um sistema de preferência mais flexível, pois não obrigam a estruturação hierárquica dos critérios nem a comparação entre as alternativas. Gomes, Araya e Cariggnano (2004) destacam que os diversos métodos desta família se diferenciam pelo tipo de problemática que se propõem a resolver, pelas informações inter e intracritérios necessárias e pela quantidade de relações de superação construídas e utilizadas.

0 método ELECTRE 11 foi desenvolvido por Roy e Bertier (1971, 1973) e pode ser considerado uma extensão do método ELECTRE 1, uma vez que necessita dos dois gráficos produzidos pelo ELECTRE 1 como dados de entrada, representando uma estrutura de preferência forte e outra fraca. O ELECTRE 11 resulta num ranking das alternativas não-dominadas, sendo indicado para a problemática de ordenação.

A idéia no ELECTRE 11 é escolher aqueles sistemas são preferidos pela maioria dos critérios e que não ultrapassem um determinado nível de desconforto ou descontentamento aceito pelo decisor, para nenhum dos critérios considerados. Existem duas medidas distintas: a de concordância e a de discordância.

O ELECTRE 11 estabelece uma ordenação completa sobre um conjunto de alternativas inicialmente consideradas que satisfaça:

- 0 teste da concordância, em que a medida da concordância está acima de um nível mínimo de aceitabilidade; e

- 0 teste da discordância, em que a medida da discordância está abaixo de um nível máximo tolerável de discordância.

As definições de concordância e discordância do ELECTRE 11 diferem daquelas apresentadas para o ELECTRE 1 (GOICOECHEA; HANSEN; DUCKSTEIN, 1982). Para poder analisar as matrizes de concordância e discordância são definidos valores de referência $p$ e $q$, pertencentes ao intervalo de variação entre 0 e 1 . Esses valores indicam que a concordância desejada deverá ser superior ou igual a $p$ e que $q$ definirá a máxima discordância tolerável.

0 procedimento de ordenação do método ELECTRE 11 constitui-se de dois estágios distintos de pré-ordenação. No primeiro estágio define-se a ordenação progressiva e, no segundo, a regressiva. As duas pré-ordenações obtidas, geralmente, são diferentes, porém próximas. Neste caso, o AD pode escolher a média entre elas, caso contrário, deve-se redefinir o problema e reaplicar o método.

Em síntese, o ELECTRE 11 busca ordenar um conjunto de alternativas da melhor para a pior, sendo desenvolvido para resolver problemas de ordenação. São utilizados os conceitos de concordância e discordância para ordenar o conjunto de alternativas. A ordenação é obtida através de duas pré-ordens construídas a partir das relações de sobreclassificação forte e fraca. 0 método ELECTRE 11 aplica-se a problemas que envolvem ordenação de alternativas.

Miranda e Almeida (2004) e Costa; Motta, Gutierrez, 2006; Costa, H. G.; Costa, J. A. B.; Caiado (2006) apresentam estudos envolvendo aplicações do ELECTRE 11. Soares de Mello et al. (2005b) 
apresentam uma aplicação do ELECTRE 1 na avaliação do tamanho dos aeroportos portugueses. Gomes (1989) realiza um estudo comparativo entre o ELECTRE 11 e o método TODIM aplicado em uma investigação sobre sistemas alternativos de transporte.

\subsection{Definição de critérios}

A escolha dos critérios, como exposto a seguir, foi efetuada de acordo com os objetivos do problema e representam os interesses e a forma de estruturar as decisões do tomador de decisão. 0 decisor assumido é fictício, dada a dificuldade de acesso aos chefes de equipe de $\mathrm{F} 1$ para discussão desta proposta.

Os critérios considerados no problema estão listados a seguir:

- C1 - Desempenho:

É um critério qualitativo que busca avaliar as performances individuais de cada piloto durante as corridas, independentemente da equipe à qual o piloto pertence. A pontuação deste critério é a soma, para cada uma das corridas da temporada, dos pontos obtidos pelos pilotos, de acordo com a Tabela 2. Por exemplo, um piloto que em uma corrida larga na sétima colocação e consegue completar a prova em sexto recebe 4 pontos.

A Tabela 3, a seguir, apresenta a valoração completa do critério C1 - Desempenho. Este critério faz uso de uma escala ordinal. Como apontado no item 4, o método ELECTRE 11 trabalha com relações ordinais.

- C2 - Pontuação do piloto no campeonato:

É um critério quantitativo que utiliza os pontos conquistados pelos pilotos na temporada em análise, conforme as regras oficiais de pontuação utilizadas no campeonato, apresentada na Tabela 1.

- C3 - Pontuação da equipe no campeonato:

Assim como no critério anterior, utiliza os pontos obtidos no campeonato pela equipe à qual o piloto pertence. Com este critério, busca-se compensar pilotos que pertenciam a equipes pequenas e médias, não possuindo, portanto, as mesmas condições de competitividade que os pilotos das equipes mais fortes. É, também, um critério quantitativo.
- C4 - Experiência:

É um critério qualitativo, que foi medido pelo ponto central da experiência do piloto. É a distância percorrida pelo piloto no número total de provas de que participou na Fórmula 1, até o final da temporada de 2007, à média do número de provas de todos os pilotos que participaram do campeonato de 2007.

Os critérios C1 - Desempenho, C2 - Pontuação do piloto no campeonato e C4 - Experiência são de maximização. Destaque-se que neste último o objetivo é favorecer os pilotos com mínima ou máxima experiência, conforme mencionado no item 2. Pilotos com distâncias pequenas à média são, teoricamente, pilotos no auge da carreira.

0 critério C3 - Pontuação da equipe no campeonato é de minimização, pois tem o objetivo de valorizar os pilotos das equipes menores.

\subsection{Peso dos critérios}

Muitas vezes, estudos que envolvem análise multicritério utilizam a técnica de aplicação de questionários ou entrevistas com o propósito de melhor traduzir no peso de cada critério ou atributo as visões de mundo do AD. Contudo, aqui, optou-se pela não utilização deste recurso, dada a grande dificuldade de acesso aos reais tomadores de decisão considerados.

0 peso adotado para os critérios selecionados neste trabalho foram estabelecidos pelos decisores que desempenham o papel de chefe de equipe por valoração direta, sendo, posteriormente, normalizados. Estes pesos estão apresentados na Tabela 4.

\subsection{Alternativas do processo decisório}

Este estudo de caso considerou como alternativas os pilotos que participaram das provas do Campeonato de 2007 da Fórmula 1, com exceção do piloto Kazuki Nakajima, que participou apenas do Grande Prêmio do Brasil, pela equipe WilliamsToyota.

Tabela 2. Valoração da performance dos pilotos.

\begin{tabular}{cl}
\hline Pontuação & \\
\hline 0 & O piloto não participou da prova \\
1 & 0 piloto não concluiu a prova \\
2 & 0 piloto concluiu a prova em uma posição pior do que a que tinha na largada \\
3 & 0 piloto manteve a posição de largada \\
4 & 0 piloto concluiu a prova em uma posição melhor do que a que tinha na largada \\
\hline
\end{tabular}


Tabela 3. Valoração do critério C1 - Desempenho.

\begin{tabular}{|c|c|c|c|c|c|c|c|c|c|c|c|c|c|c|c|c|c|c|}
\hline \multirow[b]{2}{*}{ Piloto } & \multicolumn{17}{|c|}{ Corrida } & \multirow[b]{2}{*}{$\stackrel{\pi}{\tilde{D}}$} \\
\hline & $\frac{\stackrel{\pi}{\frac{\pi}{\pi}}}{\stackrel{\frac{\pi}{2}}{\frac{Z}{2}}}$ & 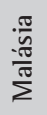 & $\frac{\frac{\Xi}{\pi}}{\frac{\pi}{\pi}}$ & $\begin{array}{l}\frac{\Xi}{\pi} \\
\text { ڤे }\end{array}$ & $\begin{array}{l}\stackrel{\mathscr{U}}{\pi} \\
\stackrel{0}{0} \\
\sum\end{array}$ & $\frac{-\pi / 0}{\pi}$ & 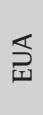 & 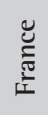 & 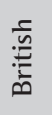 & 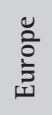 & 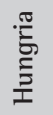 & $\underset{F}{\stackrel{\vec{D}}{\Xi}}$ & $\frac{\lambda}{ \pm}$ & $\frac{\frac{5}{\tilde{E}}}{\frac{\tilde{D}}{\tilde{D}}}$ & 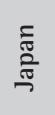 & $\stackrel{\pi}{\stackrel{\Xi}{E}}$ & $\begin{array}{l}\overline{\bar{n}} \\
\overline{\mathscr{n}} \\
\bar{D}\end{array}$ & \\
\hline Adrian Sutil & 4 & 1 & 4 & 4 & 1 & 1 & 4 & 4 & 1 & 1 & 4 & 2 & 4 & 4 & 4 & 1 & 1 & 45 \\
\hline Alexander Wurz & 1 & 4 & 4 & 1 & 4 & 4 & 4 & 4 & 4 & 4 & 4 & 4 & 4 & 1 & 1 & 4 & 0 & 52 \\
\hline Anthony Davidson & 4 & 4 & 2 & 4 & 4 & 4 & 4 & 1 & 1 & 4 & 1 & 4 & 4 & 4 & 1 & 1 & 4 & 51 \\
\hline Chistijan Albers & 1 & 1 & 4 & 4 & 4 & 1 & 4 & 1 & 4 & 0 & 0 & 0 & 0 & 0 & 0 & 0 & 0 & 24 \\
\hline David Coulthard & 1 & 1 & 1 & 4 & 4 & 1 & 1 & 4 & 4 & 4 & 4 & 4 & 1 & 1 & 4 & 2 & 4 & 45 \\
\hline Felipe Massa & 4 & 2 & 3 & 3 & 3 & 1 & 3 & 2 & 4 & 4 & 4 & 3 & 1 & 3 & 2 & 3 & 4 & 49 \\
\hline Fernando Alonso & 3 & 4 & 2 & 2 & 3 & 2 & 3 & 4 & 4 & 4 & 4 & 4 & 2 & 3 & 1 & 4 & 4 & 54 \\
\hline Giancarlo Fisichella & 4 & 4 & 2 & 4 & 4 & 1 & 4 & 2 & 4 & 4 & 4 & 4 & 4 & 1 & 4 & 4 & 1 & 55 \\
\hline Heikki Kovalainen & 4 & 4 & 4 & 4 & 4 & 4 & 4 & 4 & 4 & 4 & 4 & 4 & 2 & 4 & 4 & 4 & 1 & 64 \\
\hline Jarno Trulli & 4 & 4 & 4 & 1 & 4 & 1 & 4 & 1 & 1 & 1 & 4 & 4 & 1 & 2 & 4 & 4 & 4 & 49 \\
\hline Jenson Button & 4 & 4 & 1 & 4 & 4 & 1 & 4 & 4 & 4 & 1 & 1 & 4 & 4 & 1 & 2 & 4 & 1 & 48 \\
\hline Kimi Raikkonen & 3 & 3 & 3 & 1 & 4 & 2 & 3 & 4 & 4 & 4 & 4 & 4 & 4 & 3 & 3 & 4 & 4 & 57 \\
\hline Lewis Hamilton & 4 & 4 & 3 & 4 & 3 & 3 & 3 & 2 & 2 & 4 & 3 & 2 & 2 & 3 & 3 & 1 & 4 & 51 \\
\hline Mark Webber & 4 & 3 & 1 & 1 & 1 & 2 & 4 & 4 & 1 & 1 & 3 & 1 & 4 & 3 & 1 & 2 & 1 & 37 \\
\hline Nick Heidfeld & 2 & 4 & 4 & 1 & 4 & 4 & 1 & 4 & 4 & 2 & 2 & 4 & 2 & 4 & 2 & 4 & 3 & 52 \\
\hline Nico Rosberg & 4 & 1 & 3 & 4 & 4 & 2 & 2 & 3 & 4 & 1 & 2 & 4 & 4 & 2 & 1 & 4 & 4 & 49 \\
\hline Ralf Schumacher & 4 & 4 & 4 & 1 & 4 & 4 & 1 & 4 & 1 & 1 & 2 & 4 & 4 & 3 & 1 & 1 & 4 & 47 \\
\hline Robert Kubica & 1 & 4 & 3 & 4 & 4 & 1 & 0 & 3 & 3 & 2 & 4 & 2 & 4 & 4 & 4 & 1 & 4 & 48 \\
\hline Rubens Barrichello & 4 & 4 & 4 & 4 & 4 & 4 & 1 & 4 & 4 & 4 & 4 & 4 & 4 & 4 & 4 & 4 & 1 & 62 \\
\hline Sakon Yamamoto & 0 & 2 & 0 & 0 & 0 & 0 & 0 & 0 & 0 & 0 & 1 & 4 & 4 & 4 & 4 & 4 & 1 & 22 \\
\hline Scott Speed & 1 & 4 & 1 & 1 & 4 & 1 & 4 & 1 & 1 & 1 & 0 & 0 & 0 & 0 & 0 & 0 & 0 & 19 \\
\hline Sebastian Vettel & 0 & 2 & 0 & 0 & 0 & 0 & 2 & 0 & 0 & 0 & 4 & 4 & 4 & 1 & 1 & 4 & 1 & 21 \\
\hline Takuma Sato & 4 & 4 & 1 & 4 & 4 & 4 & 1 & 4 & 4 & 1 & 4 & 4 & 4 & 4 & 4 & 4 & 4 & 59 \\
\hline Vitantonio Liuzzi & 4 & 4 & 1 & 1 & 1 & 1 & 4 & 1 & 2 & 1 & 1 & 4 & 4 & 4 & 4 & 4 & 4 & 45 \\
\hline
\end{tabular}

Tabela 4. Peso dos critérios.

\begin{tabular}{cccc} 
Critério & Descrição & $\begin{array}{c}\text { Peso } \\
\text { atribuído }\end{array}$ & $\begin{array}{c}\text { Peso } \\
\text { normalizado }\end{array}$ \\
\hline C1 & Desempenho & 30 & 0,3 \\
C2 & Pontos dos pilotos & 20 & 0,2 \\
C3 & Pontos das equipes & 20 & 0,2 \\
C4 & Experiência & 30 & 0,3 \\
\hline
\end{tabular}

A Tabela 5 mostra a valoração das alternativas em cada um dos critérios considerados na análise para o Campeonato de 2007.

\section{Resultados}

0 primeiro resultado fornecido pelo ELECTRE 11, com os parâmetros iniciais sugeridos pelo $\mathrm{AD}$, não foi suficientemente discriminatório para estabelecer uma ordenação no conjunto das alternativas. Uma análise de sensibilidade foi feita para verificar a sensibilidade da solução a variações nos parâmetros do modelo (pesos, índices de concordância e discordância). Foram consideradas variações apenas nos índices de concordância e discordância, sendo possível, assim, obter-se um ranking das alternativas de solução do problema que gerou resultados bastante satisfatórios para o AD. Novas pequenas alterações foram realizadas, mas como houve pouca variação nos resultados encontrados, a partir dos valores de $p$ e $q$ considerados, julgou-se que o estabelecimento dos limiares $p$ e $q$ não é crítico para o modelo.

0 resultado dessas outras análises não foi apresentado por não acrescentar informações a esse estudo.

Outra análise foi realizada a partir da alteração do peso dos critérios, de modo a aumentar a importância do critério C1 - Desempenho. Contudo, os resultados obtidos não apresentaram nenhuma alteração em relação ao ranking anterior.

A ordenação obtida pelo ELECTRE 11 é mostrada na Tabela 6. 
Tabela 5. Valoração das alternativas por critério.

\begin{tabular}{lcccc}
\hline \multirow{1}{*}{ Alternativas } & \multicolumn{4}{c}{ Critérios } \\
\cline { 2 - 5 } & $\begin{array}{c}\text { Desempenho } \\
\text { (C1) }\end{array}$ & $\begin{array}{c}\text { Pontos } \\
\text { piloto } \\
\text { (C2) }\end{array}$ & $\begin{array}{c}\text { Pontos } \\
\text { equipe } \\
\text { (C3) }\end{array}$ & $\begin{array}{c}\text { Experiência } \\
\text { ponto central } \\
\text { (C4) }\end{array}$ \\
\hline Adrian Sutil & 45 & 1 & 1 & 72 \\
Alexander Wurz & 52 & 13 & 33 & 20 \\
Anthony Davidson & 51 & 0 & 4 & 69 \\
Christijan Albers & 24 & 0 & 1 & 44 \\
David Coulthard & 45 & 14 & 24 & 140 \\
Felipe Massa & 49 & 94 & 204 & 1 \\
Fernando Alonso & 54 & 109 & 218 & 16 \\
Giancarlo Fisichella & 55 & 21 & 51 & 106 \\
Heikki Kovalainen & 64 & 30 & 51 & 72 \\
Jarno Trulli & 49 & 8 & 13 & 95 \\
Jenson Button & 48 & 6 & 6 & 47 \\
Kimi Raikkonen & 57 & 110 & 204 & 33 \\
Lewis Hamilton & 51 & 109 & 218 & 72 \\
Mark Webber & 37 & 10 & 24 & 15 \\
Nick Heidfeld & 52 & 61 & 101 & 44 \\
Nick Rosberg & 49 & 20 & 33 & 54 \\
Ralf Schumacher & 47 & 5 & 13 & 91 \\
Robert Kubica & 48 & 39 & 101 & 67 \\
Rubens Barrichello & 62 & 0 & 6 & 164 \\
Sakon Yamamoto & 22 & 0 & 1 & 75 \\
Scott Speed & 19 & 0 & 8 & 61 \\
Sebastian Vettel & 21 & 5 & 8 & 81 \\
Takuma Sato & 59 & 4 & 4 & 2 \\
Vitantonio liuzzi & 45 & 3 & 8 & 50 \\
\hline \multirow{2}{*}{ (10 } & & & & \\
\hline
\end{tabular}

Tabela 6. Ordenação ELECTRE 11 e oficial.

\begin{tabular}{ll}
\hline \multicolumn{1}{c}{ Ordenação electre 11 } & \multicolumn{1}{c}{ Ordenação oficial } \\
\hline 1 - Kimi Raikkonen & 1 - Kimi Raikkonen \\
2 - Lewis Hamilton & 2 - Lewis Hamilton \\
3 - Heikki Kovalainen & 3 - Fernando Alonso \\
4 - Nick Heidfeld & 4 - Felipe Massa \\
5 - Giancarlo Fisichella & 5 - Nick Heidfeld \\
6 - Robert Kubica & 6 - Robert Kubica \\
7 - Rubens Barrichello & 7 - Heikki Kovalainen \\
8 - David Coulthard & 8 - Giancarlo Fisichella \\
9 - Nico Rosberg & 9 - Nico Rosberg \\
10 - Jarno Trulli & 10 - David Coulthard \\
11 - Fernando Alonso & 11 - Alexander Wurz \\
12 - Ralf Schumacher & 12 - Mark Webber \\
13 - Felipe Massa & 13 - Jarno Trulli \\
14 - Anthony Davidson & 14 - Sebastian Vettel \\
15 - Scott Speed & 15 - Jenson Button \\
16 - Alexander Wurz & 16 - Ralf Schumacher \\
17 - Jenson Button & 17 - Takuma Sato \\
18 - Mark Webber & 18 - Vitantonio Liuzzi \\
19 - Vitantonio Liuzzi & 19 - Adrian Sutil \\
20 - Adrian Sutil & 20 - Rubens Barrichello \\
21 - Takuma Sato & 21 - Scott Speed \\
22 - Sebastian Vettel & 22 - Anthony Davidson \\
23 - Christijan Albers & 23 - Sakon Yamamoto \\
24 - Sakon Yamamoto & 24 - Christijan Albers \\
\hline
\end{tabular}

\subsection{Análise comparativa com o método ordinal Condorcet}

Uma vez que a análise de sensibilidade não apresentou variação significativa, optou-se pela comparação dos resultados encontrados pelo ELECTRE $11 \mathrm{com}$ os de outro método multicritério ordinal. 0 método de Condorcet foi escolhido por ser um método bastante simples, de fácil entendimento por parte do $A D$ e que exige ainda menos do $A D$ que o ELECTRE 11.

0 método de Condorcet, idealizado por Jean-Marie Antoine Nicolas de Caritat, Marquês de Condorcet (1743-1794), é considerado precursor da atual escola francesa de multicritério e trabalha com relações de superação. As alternativas são comparadas sempre duas a duas e constrói-se um grafo que expressa a relação entre elas (BOAVENTURA NETO, 2003). Este método tem a vantagem de impedir distorções ao fazer com que a posição relativa de duas alternativas independa de suas posições relativas a qualquer outra. No entanto, pode conduzir ao chamado paradoxo de Condorcet, ou situação de intransitividade. Isso acontece quando a alternativa $\mathrm{A}$ supera a alternativa $B$, que supera a $C$, que por sua vez supera a alternativa A (Figura 1). Esta situação pode ser aproveitada em certos problemas, quando o objetivo é agrupar alternativas (SOARES DE MELLO et al., 2005). No entanto, quando ocorre, impossibilita gerar uma ordenação das alternativas. Quando os ciclos de intransitividade não aparecem e deseja-se obter uma pré-ordem total, o método de Condorcet deve ser preferido ao de Borda (SOARES DE MELLO et al., 2004). Se o objetivo for realizar uma escolha, mesmo com intransitividades, o método de Condorcet tem uma vantagem: obriga a intervenções interativas com o decisor, evitando o paradigma do ótimo. Este paradigma é criticado por Climaco (2003).

0 método de Copeland usa a mesma matriz de adjacência que representa o grafo do método de Condorcet. A partir dela calcula-se a soma das vitórias menos as derrotas, em uma votação por

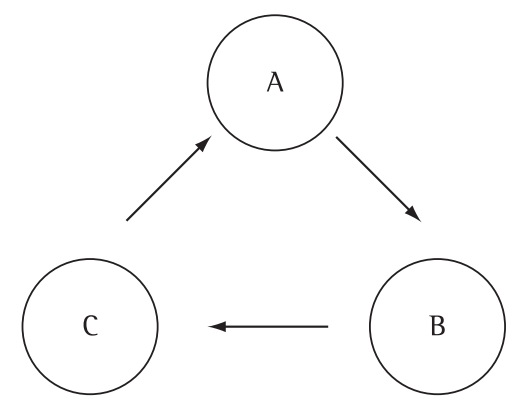

Figura 1. Tripleta de Condorcet. 
Tabela 7. Matriz de adjacência de Condorcet.

\begin{tabular}{|c|c|c|c|c|c|c|c|c|c|c|c|c|c|c|c|c|c|c|c|c|c|c|c|c|}
\hline & 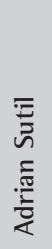 & 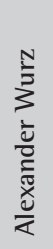 & 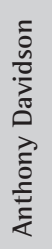 & 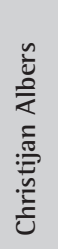 & 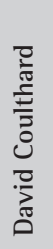 & 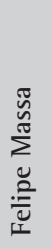 & 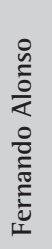 & 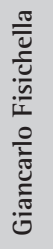 & 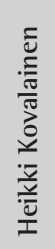 & 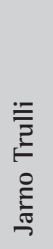 & 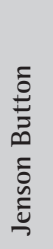 & 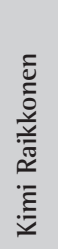 & 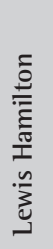 & 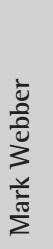 & 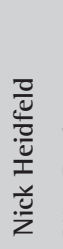 & 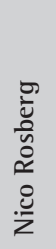 & 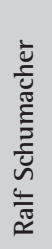 & $\begin{array}{l}\frac{\pi}{0} \\
\frac{0}{2} \\
\frac{\pi}{2} \\
\frac{0}{0} \\
\cong\end{array}$ & 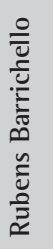 & 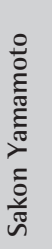 & 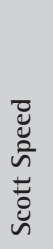 & 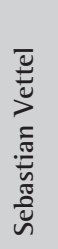 & 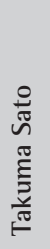 & 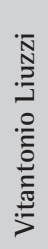 \\
\hline Adrian Sutil & & & 1 & 1 & & & & & & & & & & 1 & & & & & & 1 & 1 & & & 1 \\
\hline Alexander Wurz & 1 & & 1 & 1 & 1 & & & & & 1 & 1 & & & 1 & & 1 & 1 & & 1 & 1 & 1 & 1 & 1 & 1 \\
\hline Anthony Davidson & 1 & & & 1 & & & & & & & 1 & & & 1 & & 1 & & & & 1 & 1 & & 1 & 1 \\
\hline Christijan Albers & & & & & & & & & & & & & & & & & & & & 1 & 1 & & & \\
\hline David Coulthard & 1 & 1 & 1 & 1 & & & & & & 1 & 1 & & & 1 & & & 1 & & 1 & 1 & 1 & 1 & 1 & 1 \\
\hline Felipe Massa & 1 & 1 & 1 & 1 & 1 & & & 1 & & 1 & 1 & & & 1 & 1 & 1 & 1 & 1 & 1 & 1 & 1 & 1 & 1 & 1 \\
\hline Fernando Alonso & 1 & 1 & 1 & 1 & 1 & & & 1 & 1 & 1 & 1 & & 1 & 1 & 1 & 1 & 1 & 1 & 1 & 1 & 1 & 1 & 1 & 1 \\
\hline Giancarlo Fisichella & 1 & 1 & 1 & 1 & 1 & 1 & 1 & & 1 & 1 & 1 & & 1 & 1 & 1 & 1 & 1 & 1 & 1 & 1 & 1 & 1 & 1 & 1 \\
\hline Heikki Kovala & 1 & 1 & 1 & 1 & 1 & 1 & 1 & 1 & & 1 & 1 & 1 & 1 & 1 & 1 & 1 & 1 & 1 & 1 & 1 & 1 & 1 & 1 & 1 \\
\hline Jarno Trulli & 1 & & 1 & 1 & & & & & & & 1 & & & 1 & & 1 & 1 & 1 & 1 & 1 & 1 & 1 & 1 & 1 \\
\hline Jenson Button & 1 & & 1 & 1 & & & & & & & & & & 1 & & & 1 & & 1 & & 1 & 1 & 1 & 1 \\
\hline Kimi & 1 & 1 & 1 & 1 & 1 & 1 & 1 & 1 & 1 & 1 & 1 & & 1 & 1 & 1 & 1 & 1 & 1 & 1 & 1 & 1 & 1 & 1 & 1 \\
\hline Lewis & 1 & 1 & 1 & 1 & 1 & 1 & & 1 & 1 & 1 & 1 & 1 & & 1 & 1 & 1 & 1 & 1 & 1 & 1 & 1 & 1 & 1 & 1 \\
\hline Mark Webber & 1 & & 1 & 1 & & & & & & 1 & 1 & & & & & & 1 & & 1 & 1 & 1 & 1 & 1 & 1 \\
\hline Nick Heidfeld & 1 & 1 & 1 & 1 & 1 & 1 & & 1 & 1 & 1 & 1 & & & 1 & & 1 & 1 & 1 & 1 & 1 & 1 & 1 & 1 & 1 \\
\hline Nico Rosberg & 1 & 1 & 1 & 1 & 1 & & & & & 1 & 1 & & & 1 & & & & & 1 & 1 & 1 & 1 & 1 & 1 \\
\hline Ralf Schumacher & 1 & & 1 & 1 & & & & & & & 1 & & & 1 & & & & & 1 & 1 & 1 & 1 & 1 & 1 \\
\hline Robert Kubica & 1 & 1 & 1 & 1 & 1 & & & 1 & 1 & 1 & 1 & & & 1 & & 1 & 1 & & 1 & 1 & 1 & 1 & 1 & 1 \\
\hline Rubens Barrichello & 1 & 1 & 1 & 1 & 1 & 1 & 1 & 1 & & 1 & 1 & 1 & 1 & 1 & & 1 & 1 & 1 & & 1 & 1 & 1 & 1 & 1 \\
\hline Sakon Yamamoto & & & & & & & & & & & 1 & & & & & & & & & & 1 & & & \\
\hline Scott Speed & & & & 1 & & & & & & & 1 & & & & & & & & & & & & 1 & 1 \\
\hline Sebastian Vettel & 1 & & 1 & 1 & & & & & & & 1 & & & & & & & & 1 & 1 & 1 & & 1 & 1 \\
\hline Takuma Sato & 1 & & 1 & 1 & & 1 & & & & & & & & & & & & & & 1 & 1 & & & 1 \\
\hline Vitantonio Liuzzi & 1 & & 1 & 1 & & & & & & & 1 & & & 1 & & & & & 1 & 1 & 1 & & 1 & \\
\hline
\end{tabular}

maioria simples. As alternativas são então ordenadas pelo resultado dessa soma. 0 método de Copeland alia a vantagem de sempre fornecer uma ordenação total, ao contrário do de Condorcet, ao fato de dar o mesmo resultado do de Condorcet quando não apresenta nenhum ciclo de intransitividade. Quando esses ciclos existem, o método de Copeland permite fazer a ordenação e mantém a ordenação das alternativas que não pertencem a nenhum ciclo de intransitividade. Apesar de computacionalmente mais exigente que Borda, quando há necessidade de estabelecer uma relação de pré-ordem, ou ordem lato sensu, este método fornece sempre uma resposta (ao contrário do método de Condorcet) e, apesar de não eliminar, reduz bastante a influência de alternativas irrelevantes.

\subsubsection{Resultados obtidos com o método ordinal Condorcet}

Os dados da Tabela 5 são usados para construir o grafo de Condorcet, cuja matriz de adjacência é apresentada na Tabela 7.
A aplicação da técnica de destilação descendente, como explicada em Soares de Mello et al. (2005), mostra a existência de seis ciclos de intransitividade. 0 primeiro engloba os pilotos Kimi Raikkonen e Heikki Kovalainen. Em seguida, os pilotos Fernando Alonso, Lewis Hamilton e Rubens Barrichello. 0 terceiro ciclo é composto pelos pilotos Felipe Massa e Nick Heidfeld. 0 quarto ciclo envolve os pilotos Alexander Wurz e Nico Rosberg. 0 quinto ciclo de intransitividade é formado pelos pilotos Jarno Trulli e Mark Webber. Finalmente, os pilotos Jenson Button e Takuma Sato integram o sexto ciclo. Não foi necessária a utilização da técnica de destilação ascendente por ela apresentar os mesmos resultados, mantendo os mesmos ciclos de intransitividade.

Deve-se enfatizar que os pilotos pertencentes ao mesmo ciclo não estão empatados. 0 ciclo caracteriza uma situação que não segue a racionalidade. Por esse motivo é impossivel saber se estão empatados ou se há alguma ordem. Num método que conduza a ciclos há ausência de relações binárias de ordem ou pré-ordem. 
Tabela 8. Ordenação segundo o método de Condorcet.

\begin{tabular}{cc}
\hline Ordenação & Piloto \\
\hline 1 & Kimi Raikkonen \\
2 & Heikki Kovalainen \\
3 & Giancarlo Fisichella \\
4 & Fernando Alonso \\
5 & Lewis Hamilton \\
6 & Rubens Barrichello \\
7 & Nick Heidfeld \\
8 & Felipe Massa \\
9 & Robert Kubica \\
10 & Alexander Wurz \\
11 & Nico Rosberg \\
12 & David Coulthard \\
13 & Jarno Trulli \\
14 & Mark Webber \\
15 & Ralf Schumacher \\
16 & Sebastian Vettel \\
17 & Anthony Davidson \\
18 & Vitantonio Liuzzi \\
19 & Jenson Button \\
20 & Takuma Sato \\
21 & Adrian Sutil \\
22 & Christijan Albers \\
23 & Sakon Yamamoto \\
24 & Scott Speed \\
\hline
\end{tabular}

Uma vez que não é possível fazer a ordenação pelo método de Condorcet, recorreu-se ao método de Copeland. Este método, como já mencionado, utiliza a mesma matriz gerada pelo método de Condorcet - conta-se o número 1 na linha do elemento que se quer avaliar e subtrai-se 1 do total da respectiva coluna. A ordenação é feita de acordo com quem obtiver a maior pontuação, seguindo ordem decrescente. A Tabela 8 apresenta a ordenação obtida com a utilização deste método.

A alteração dos resultados no método de Condorcet apresentou alternativas consideradas desfavoráveis pelo $\mathrm{AD}$, como, por exemplo, os pilotos Fernando Alonso e Lewis Hamilton como bons pilotos para contratação por equipes pequenas ambos são pilotos consagrados e com altos salários na categoria, deixando clara a importância de se atribuir pesos diferentes para os critérios.

\section{Conclusões}

Analisando os resultados encontrados, pode-se observar que existem muitas diferenças entre a ordenação do ELECTRE 11 e a ordenação final da temporada, segundo os critérios oficiais de pontuação. Vale ressaltar que isto ocorre porque a óptica de avaliação utilizada é completamente diferente dos critérios de pontuação do campeonato, que pontua apenas os 8 primeiros pilotos classificados entre os 22 que participaram de cada prova da temporada de 2007. 0 enfoque deste trabalho é avaliar exatamente os pilotos que não têm destaque neste sistema de avaliação. Neste contexto, o método e os critérios utilizados apresentaram resultados bastante satisfatórios.

Em relação aos dois primeiros pilotos da ordenação do ELECTRE ll, Kimi Raikkonen e Lewis Hamilton, apesar de serem, respectivamente, campeão e vice-campeão da temporada de 2007, são pilotos com excelente desempenho e, no caso do piloto Lewis Hamilton, pouquíssima experiência, já que ele iniciou sua carreira na F1 no início da temporada de 2007. Entretanto, são os pilotos com maior destaque ao final da temporada e, sendo assim, não satisfazem o objetivo aqui proposto, pois recebem altos salários.

No entanto, os pilotos nas classificações seguintes se conformam bem ao perfil estipulado neste trabalho. Sendo assim, são boas opções de contratação por equipes menores: Heikki Kovalainen, Nick Heidfeld, Robert Kubica, Giancarlo Fisichella, Rubens Barrichello e David Coulthard. Os três primeiros são pilotos com pouca experiência na categoria mas que se mostraram bastante talentosos e competitivos. Os três seguintes são pilotos com bastante experiência na $\mathrm{F} 1$, que não disputam posições de destaque e têm conhecimento suficiente para desenvolver, junto com a equipe, carros bastante competitivos. É possível observar que, como esperado, essas alternativas foram apontadas como as escolhas mais indicadas.

Em relação às últimas posições da ordenação do ELECTRE 11, nota-se que os pilotos Sebastian Vettel, Christijan Albers e Sakon Yamamoto são aqueles que pertenceram às menores equipes da categoria $e$ tiveram, ainda, os piores resultados na temporada.

Deve ser observada a situação singular que o piloto Sebastian Vettel apresentou no campeonato. 0 piloto participou de oito corridas na temporada de 2007. Entretanto, sua primeira participação foi no Grande Prêmio dos EUA, pela equipe BMW, substituindo o piloto Robert Kubica, que havia se acidentado no GP do Canadá e estava impossibilitado de participar daquela prova. Nesta corrida, Vettel largou em $7^{\circ}$ e completou a prova na $8^{a}$ posição, marcando 1 ponto no campeonato. 0 piloto voltou ao campeonato a partir da 11 $1^{\text {a }}$ etapa, no GP da Hungria, pela equipe Toro Rosso, correndo até o final da temporada. Por esta equipe, o piloto conseguiu um $4^{\circ}$ lugar no GP da China, marcando mais 5 pontos no campeonato. No entanto, Vettel não conseguiu boa colocação 
na ordenação ELECTRE 11 por ter poucos pontos no critério desempenho, visto que participou de poucas provas. Além disso, neste trabalho, foi considerada apenas a sua participação pela equipe Toro Rosso.

0 ELECTRE 11 , que é de fato indicado para a problemática de ordenação, mostrou ser realmente uma metodologia adequada ao estudo realizado. Com ele foi possivel obter um ranking das alternativas de solução do problema.

Cabe ressaltar que fatores subjetivos como: preparo físico, dedicação, conhecimento de motores, entre outros, não foram incluídos no estudo, devido à dificuldade de obtenção de dados.

Como trabalhos futuros, sugere-se uma análise mais completa do tema, buscando-se pilotos em categorias de acesso à Fórmula 1, bem como a utilização de novos critérios que possam ser relevantes para os objetivos do trabalho. É indicado, ainda, o estudo de outras metodologias multicritério para estabelecer a ordenação dos pilotos, de acordo com a proposta deste trabalho.

Estudos desta natureza tornam-se mais importantes a partir da decisão da FIA, anunciada em março de 2009, ou seja, às vésperas de começar o Campeonato Mundial de 2009, de considerar apenas o número de corridas ganhas em uma temporada para determinar o campeão do mundo. Não levando em consideração os demais resultados obtidos são necessários outros métodos para identificar pilotos promissores.

\section{Referências}

ALMEIDA, A. T.; COSTA, A. P. C. S. Aplicações multicritério de apoio à decisão. Recife: UFPE, 2003.

ARROW, K. J. Social choice and individual values. New York: John Wiley and Sons, 1951.

BARBA-ROMERO, S.; POMEROL, J. C. Decisiones multicritério: fundamentos teóricos y utilizacion prática. Espanha: Universidade de Alcacá, 1997.

BOAVENTURA NETO, P. 0. Grafos: teoria, modelos, algoritmos. São Paulo: Edgard Blücher, 2003.

CliMACO, J. C. N. A critical reflection on optimal decision. European Journal of Operational Research, v. 153, n. 2, p. 506-516, 2003.

CHURILOV, L.; FLITMAN, A. Towards fair ranking of olympics achievements: the case of Sydney 2000. Computers and Operations Research, v. 33, n. 7, p. 2057-2082, 2006.

COSTA, H. G.; MOTTA, S. S.; GUTIERREZ, R. H. Avaliação da produção docente: abordagem multicritério pelo método ELECTRE 11. In: ENCONTRO NACIONAL DE ENGENHARIA DE PRODUÇÃO - ENEGEP, 26, 2006. Anais..

COSTA, H. G.; COSTA, J. A. B.; CAIADO, J. R. C. Avaliação de eqüinos "Mangalarga Marchador": uma análise multicritério pelo método ELECTRE 11. Revista Pesquisa e Desenvolvimento em Engenharia de Produção, n. 5, p. 1-17, 2006.
DIXON, M. J.; COLES, S. G. Modelling association football scores and inefficiencies in the football betting market. Journal of the Royal Statistical Society. Series C: Applied Statistics, v. 46, n. 2, p. 265-280, 1997.

DIXON, M. J. B.; ROBINSON, M. E. A. A birth process model for association football matches. Journal of the Royal Statistical Society. Series D: The Statistician, v. 47, n. 3, p. 523-538, 1998.

FIGUEIRA, J. R. A.; ROY, B. A note on the paper, "ranking irregularities when evaluating alternatives by using some ELECTRE methods", by Wang and Triantaphyllou. Omega, v. 37, n. 3, p. 731-733, 2009.

GOICOECHEA, A.; HANSEN, D.; DUCKSTEIN, L. Multiobjective decision analysis with engeneering and bussiness applications. London: John Wiley and Sons, 1982.

GOMES, L. F. A. M. Comparing two methods for multicriteria ranking of urban transportation system alternatives. Journal of Advanced Transportation, v. 23, n. 2-3, p. 217-219, 1989.

GOMES, L. F. A. M.; ARAYA, M. C. G.; CARIGGNANO, C. Tomada de decisões em cenários complexos. São Paulo: Thomson, 2004.

GOMES JÚNIOR, S. F. Métodos não convencionais de restrições aos pesos em dea, aplicados ao campeonato mundial de Fórmula 1. Niterói, 2006. Tese (Mestrado em Engenharia de Produção) -Universidade Federal Fluminense - UFF.

GOMES JÚNIOR, S. F.; SOARES DE MELLO, J. C. C. B. S. Emprego de métodos ordinais multicritério na análise do campeonato mundial de Fórmula 1. In: SIMPÓSIO DE PESOUISA OPERACIONAL E LOGÍSTICA DA MARINHA SPOLM, 10, 2007a. Anais...

GOMES JÚNIOR, S. F.; SOARES DE MELLO, J. C. C. B. S. Avaliação dos pilotos no campeonato mundial de Fórmula 1 no ano de 2006 utilizando modelo DEA com restrições cone rattio não arquimedianas. Sistemas e Gestão, v. 2, n. 3 , p. $216-230,2007 b$.

HELD, L.; VOLLNHALS, R. Dynamic rating of European football teams. IMA Journal of Management Mathematics, v. 16, n. 2, p. 121-130, 2005.

HOLDER, R. L.; NEVILL, A. M. Modelling performance at international tennis and golf tournaments: ls there a home advantage? Journal of the Royal Statistical Society Series D: The Statistician, v. 46, n. 4, p. 551-559, 1997.

JENKINS, M. A.; FLOYD, S. B. Trajectories in the evolution of technology: a multi-level study of competition in formula 1 racing. Organization Studies, v. 22, n. 6, p. 945-969, 2001.

KLADROBA, A. The problem of aggregation arising in the process of building rankings: some remarks with the example of the Formula 1 championship 1998. Jahrbucher fur Nationalokonomie und Statistik, v. 220, n. 3, p. 302-314, 2000 .

KONING, R. H. Balance in competition in dutch soccer. Journal of the Royal Statistical Society Series D: The Statistician, v. 49. n. 3, p. 419-431, 2000.

LINS, M. P. et al. Olympic ranking based on a zero sum gains DEA model. European Journal of Operational Research, v. 148 , n. 2, p. 312-322, 2003.

MIRANDA, C. M. G.; ALMEIDA, A. T. Visão multicritério da avaliação de programas de pós-graduação pela CAPES: o caso da área engenharia 111 baseado nos métodos ELECTRE 11 e MAUT. Gestão e Produção, v. 11, n. 1, p. 51-64, 2004. 
POLLARD, R. Evidence of a reduced home advantage when a team moves to a new stadium. Journal of Sports Sciences, v. 20, n. 12, p. 969-973, 2002.

ROY, B. Classement et choix en presence de points de vue multiples (la methode ELECTRE). Revue d'Informa-tique et de recherché opérationelle, v. 6, n. 8, p. 57-75, 1968.

ROY, B.; BERTIER, P. La méthode ELECTRE II. Paris: SEMA-METRA, 1971.

ROY, B.; BERTIER, P. La méthode ELECTRE Il: une application au média-planning. In: ROSS, M. (Ed.). Amsterdam: North-Holland Publishing Company, 1973. p. 291-302.

SOARES DE MELLO, M. H. C. S.; QUINTELLA, H. L. M. M.; MELLO, J. C. C. B. S. Avaliação do desempenho de alunos considerando classificações obtidas e opiniões dos docentes. Investigação Operacional, v. 24, n. 2, p. 187-196, 2004.

SOARES DE MELLO, J. C. C. B. S.; ANGULO-MEZA, L.; SILVA, B. P. B. A ranking for the olympic games with unitary input DEA models. IMA Journal of Management Mathematics, v. 20, n. 2, p. 201-211, 2009.

SOARES DE MELLO, J. C. C. B. S. et al. Use of ordinal multicriteria methods in the analysis of the Formula 1 world championship. Cadernos EBAPE, v. 3, n. 2, p. 3, 2005 a.

SOARES DE MELLO, J. C. C. B. S. et al. Avaliação do tamanho de aeroportos portugueses com relações multicritério de superação. Pesquisa Operacional, v. 25, n. 3, p. 313-330, 2005b.

SOARES DE MELlO, J. C. C. B. S. et al. Cross evaluation using weight restriction in unitary input DEA models: Theoretical aspects and application to olympic games ranking. WSEAS Transactions on Systems, v. 7, n. 1, p. 31-39, 2008.

WANG, X. Triantaphyllou: ranking irregularities when evaluating alternatives by using some ELECTRE methods. Omega, v. 36, n. 1, p. 45-63, 2008.

\section{ELECTRE 11 multicriteria method to evaluate Formula 1 world championship drivers}

\section{Abstract}

Choosing a Formula One driver is a difficult task, which goes beyond the identification of ability and courage to drive cars. Drivers that do not fight for the first places in competitions normally gain little coverage because sports broadcasts favor the winners. The search for good drivers is especially important for smaller teams that are unable to pay high salaries. Hiring a good driver may represent significant earnings, allowing for a better team performance in the championship. This article proposes a methodology to evaluate these drivers in the F1 championship using the ELECTRE 11 decision support method. The main goal is to offer hiring options for the small/medium teams, allowing them to be more competitive in the following season. The analysis was made considering the pilots of the 2007 season.

\section{Keywords}

ELECTRE 11. Multicriteria. Formula 1. 